\title{
The role of beliefs and practices in health on patients' compliance
}

\author{
Palompon R. Daisy \\ Faculty of Cebu Normal University, College of Nursing, Philippines.
}

Accepted 24 March, 2013

\begin{abstract}
The degree of influence of traditional beliefs and practices of a person as part of his or her cultural background is indeed a creative molder of a person's behavior such as attitudes, beliefs and practices regarding health promotion and prevention of illness. This study determined the correlation between health beliefs and practices and the compliance with discharge instructions, particularly on home medications and out-patient referral by patients in selected hospitals in Sogod, Southern Leyte, Philippines. Health beliefs and practices were categorized as traditional, scientific and parochial. Utilizing a correlational design, thirty respondents were selected by purposive sampling technique. A researcher-made interview schedule was utilized. The compliance behavior of the respondents on medications showed that majority of the respondents was highly and moderately compliant while compliance with out-patient referral showed that majority have poor compliance. Majority of the health beliefs most frequently identified were scientific while practices were mostly parochial. There was no significant relationship between health beliefs and practices with medication compliance. Significant correlations were shown between health beliefs and practices and out-patient referral compliance. Reasons for non-compliance for medications include financial constraints and forgetfulness while on out-patient referral, respondents did not comply the follow-up consultation, since they do not feel any symptoms anymore or went to a faith healer for further management. It is concluded that health beliefs and practices do not influence compliance of medication since it is basically a consideration of individual's budgetary capacity. It is further concluded that health beliefs and practices significantly influence out-patient referral compliance since it is affected more on behavioral attributes.
\end{abstract}

Key words: Traditional beliefs, behavior, practices, out-patient.

\section{INTRODUCTION}

Every person has his own beliefs and practices which influence his behaviors, including health-related behaviors. The Filipino culture is rich with socio-cultural beliefs and practices that often pose a challenge to health care workers like nurses (Kaplan, 1993). Their unique health beliefs and practices often contradict the scientific ways of dealing with illnesses. Hence, this study aims to determine whether individual's health beliefs and practices of clients influenced their compliance of the discharge instructions given after discharged from the hospital. Traditional health beliefs and practices have significant influence on the health of the community. According to
Morisky, (2002.), explained that these health beliefs and practices may be classified into beliefs that result in no harmful health effects, beliefs that may produce beneficial health outcomes, and beliefs and traditions which have serious, harmful health outcomes. It is on the last category that one tends to avoid as this can have serious implications in the general health of the society.

The impact of an individual's cultural influences on traditional health beliefs and practices has been reflected in a survey in Southern Leyte, Philippines which revealed that most deaths occur not in the hospital but in their homes. Out of those who died in the hospital, only few 
have no experience of consultation with faith healers or quackdoctors (herbolario), but a great majority had previous treatment from faith healers or quackdoctors before they were admitted at the hospital. Moreover, of those who died in their homes, more than half were under the care of faith healer before they died (Mosot, March to August, 1985).

The degree of influence of traditional beliefs and practices of a person as part of his or her cultural background is indeed a creative molder of a person's behavior such as the values, attitudes, beliefs and practices regarding health promotion and prevention of illness. The culture influence health and healing behaviors (Vaughn, Jacquez and Baker, 2009). It is ironical that with the onset of modernized facilities and approaches on managing diseases, there are still a number of people who refuse to accept treatment regimen and practices provided (Panopio, et al 1995). Health beliefs and practices in most cases are categorized into scientific and non-scientific. However, there are practices which are considered traditional or parochial, hence in this study, the said categories were adopted.

Health care providers experienced difficulties accepting traditional beliefs and practices among clients since most of these beliefs and practices have no scientific bases. Yet, Berger (1992) states that one's health beliefs and practices may have relevant roles in uplifting the ill person's health status, as they often are called, "faith healing" in which it is believed that a person's faith helped him recover from illness or his faith in a person who has divine powers to heal paved the way for healing. Hence, this study investigated the influence of health beliefs and practices of patients recently discharged from hospital on their compliance of the prescribed discharged instructions for follow up home care.

It is hypothesized that patient's health beliefs and practices affect the compliance of patients on the discharged instructions prescribed to him/her after discharge from the hospital, particularly on home medications and outpatient referral. Compliance of medications at home plays a very relevant role in the recovery of the patient, while out-patient referral, referring to the compliance of the patient for follow-up check-up in the clinics, provides the monitoring of the patient's health progress after hospitalization.

\section{METHODOLOGY}

This study was done in three selected secondary hospitals in Southern Leyte, Philippines, a rural area. This locale was selected as it is assumed that rural areas are more exposed to traditional health beliefs and practices than in urbanized area. Utilizing a correlational design, thirty (30) respondents were included in the study selected by purposive sampling technique. The study respondents were selected based on the criteria that they have been previously hospitalized for three days within the year at which data collection took place and at least 15 to 70 years old. In the data gathering, a researcher-made interview schedule was utilized with two parts, such as seven questions relating to patient's personal data and eight questions pertaining to the problem. Considering that the tool used was not standardized, a pilot-testing was done to five patients who were excluded from the actual sample, which enabled the researcher to improve the interview schedule and master the skills in interviewing.

During the actual interview, respondents were allowed to express their answers during the interview and the transcription of interview proceedings was done. From the transcribed interview, the researcher noted the beliefs and practices identified by the respondents. The health beliefs and practices were categorized as traditional, parochial and scientific. Variables on compliance with discharge instructions on medication and out-patient referrals were categorized into high, moderate and poor compliance. Respondents' health beliefs and practices were also classified as traditional, parochial and scientific. Traditional beliefs and practices are those which were known and practiced as they were handed down from their ancestors, and did not vary in concepts and practice; parochial on the other hand, are beliefs and practices which are known in a particular place but varies in the method of application, and scientific are those which are supported with medical explanations. In classifying the health beliefs and practices, the researcher hired two social sciences expert to categorize the responses of the respondents, and an inter-rater reliability testing was done which had score of 0.9 , which is very high. Data were analyzed using percentage and chi-square in which the level of significance was set at 0.05 .

\section{RESULTS AND DISCUSSION}

\section{Compliance of discharge Instructions}

Study findings revealed that in terms of compliance of discharge instructions (Table 1), majority of the respondents were either highly or moderately compliant with home medications, while majority were poorly compliant with out-patient referral. The compliance on home medications indicate that some respondents have completed their therapy while others did not due to reasons which they have identified as forgetfulness, financial constraints or advised by faith healer to stop taking the medications. However, an important observation was the poor compliance on out-patient referral which the respondents reasoned out that they lack money to pay for consultation and no time in going to the clinic for follow-up check-up. The findings seemed alarming since referral for consultation after being discharged from hospitalization indicates the necessity for prevention of recurrence of illness or complications.

The economic implication for non-compliance of outpatient referral can be resolved by referring clients to the health center since these facilities do not require payment for consultation.

\section{Categorization of health beliefs and practices}

Utilizing open-ended, multiple response data retrieval, 
Table 1. Extent of compliance on discharge instructions $(n=30)$.

\begin{tabular}{lcc}
\hline Level of compliance & Home medications (\%) & Out-patient referral (\%) \\
\hline High & 46.66 & 30 \\
Moderate & 46.66 & 26.66 \\
Poor & 6.66 & 43.33 \\
\hline
\end{tabular}

Table 2. Health beliefs categorization.

\begin{tabular}{lc}
\hline Health belief & Frequency $^{*}$ \\
\hline Traditional & 5 \\
Illness is due to exposure to wind (panuhot or ayri) & 3 \\
Illness is given by dead relatives (gihangop sa kalag) & 2 \\
Illness is due to taking a bath every night & 1 \\
Illness is due to curse (gaba) & 1 \\
Illness is due to exposure to draft (tun-og) & 1 \\
Illness was due to a relapse (bughat) & 1 \\
Gastrointestinal neurosis (gikabuhi) & \\
Parochial & 3 \\
Illness if due to witchcraft ( barang) & 1 \\
Illness was given by a supernatural being who was attracted to the patient (gikaibugan ug dili ingon nato) & 1 \\
Sleeping under the stairs ( puus) & 1 \\
Has heart illness due to family separation & 1 \\
Became ill due to supernatural spirit in water (gibuyagan) & \\
Scientific & 8 \\
Illness is due to over-fatigue (hago) & 2 \\
Diabetes is caused by high sugar intake, obesity and heredity & 2 \\
Acidic/sour fruits cause ulcer or gastritis & 2 \\
Ulcer or gastritis is due to skipping of meals & 1 \\
Severity of illness is due to taking a bath even with fever & 1 \\
Skins itchiness is due to allergic reaction to cold & 1 \\
Hypertension is due to heredity & 1 \\
Diarrhea is due to indigestions (wala kahilisi) & 1 \\
Asthma attack was due to smell of soil when raining & 1 \\
Cough was developed due to exposure to rain & 1 \\
Hypertension is caused by high salt intake & 1 \\
Tuberculosis was due to inadequate nutrition & 1 \\
Cardiac problem was due to tension & 1 \\
Hypertension is caused by overexposure to sunlight & 1 \\
Hypertension is caused by high cholesterol & 1 \\
\hline
\end{tabular}

*Open-ended, multiple response.

Table 2 revealed that majority of the health beliefs most frequently identified were scientific such as illness is due to fatigue, diabetes is caused by obesity and heredity, ulcer or gastritis is due to skipping of meals among others. Some of the mostly identified traditional beliefs were illness is due to exposure to air (panuhot), it is given by dead relatives (gihangop sa kalag), illness is due to taking a bath at night time or it is due to curse or gaba. 
Table 3. Health practices categorization.

\begin{tabular}{|c|c|}
\hline Health belief & Frequency $^{\star}$ \\
\hline \multicolumn{2}{|l|}{ Traditional } \\
\hline Offered a mass for the dead. & 2 \\
\hline Offered a novena or rosary to the Virgin Mary & 2 \\
\hline Drinking warm beverage to cure Gastrointestinal neurosis ( kabuhi) & 1 \\
\hline \multicolumn{2}{|l|}{ Parochial } \\
\hline Drinking boiled parts of Nino plants & 8 \\
\hline Healing through whispering, blowing or putting saliva (tayhop) & 7 \\
\hline Applying oil or water blessed by faith healer or herbolario/local quackdoctor ( banyus) & 7 \\
\hline Drinking of boiled parts (laga) of unidentified trees or plants & 5 \\
\hline Submit to care of faith healer or herbolario & 4 \\
\hline Reflexology & 4 \\
\hline Applying (haklap) leaves on the skin & 3 \\
\hline Guessing ritual (patigui) to determine if illness is given by dead souls & 3 \\
\hline Smoking ritual (palina) & 3 \\
\hline Applying kerosene on the skin as liniment & 2 \\
\hline Drinking of water or oil blessed by faith healer & 2 \\
\hline Palpating radial pulse to diagnose illness (himulso) & 2 \\
\hline Steaming ritual ( tuob) & 2 \\
\hline Applying Nino juicen on affected skin (haplas) & 2 \\
\hline Wearing amulet or charm (habak) & 2 \\
\hline Ritual (pabuhat) to cure illness given by dead souls & 2 \\
\hline Applying Nino leaves on skin (haklap) & 1 \\
\hline Making oil ( lana) from a coconut tree with only one fruit & 1 \\
\hline Avoidance of cursing (tunglo) to avoid illness & 1 \\
\hline Letting the herbolario offer prayers (orasyon) & 1 \\
\hline \multicolumn{2}{|l|}{ Scientific } \\
\hline Applying (mamanyus) liniment such as efficascent oil, or Omega & 11 \\
\hline Massage (hilot) of affected body parts & 10 \\
\hline Drinking boilded (nilaga) acceptable herbs & 8 \\
\hline Applying herbal leaves on the skin & 5 \\
\hline Drinking lots of water to cure urinary infection & 4 \\
\hline Washing the feet with boiled herbs & 1 \\
\hline Eating garlic to reduce hypertension & 1 \\
\hline Drinking honey as supplement & 1 \\
\hline Drinking young coconut juice as diuretics & 1 \\
\hline
\end{tabular}

*Open-ended, multiple response

This implies that although the respondents have their animistic beliefs, most of the beliefs are scientifically related which increases the possibility of promoting health or early recovery. This result is supported by a study of Neff (1998) as cited by Kemp (2005) that folk illnesses are health problems which are diagnosed, interpreted in the light of ones culture of healing associated with psychological and religious color.
On the health practices, Table 3 revealed that there is a high frequency of practice categorized as parochial health practices, which imply the need to spread the acceptable health practices which are scientifically proven. Although the challenge of changing the perceptions of the community on these common practices they have been used to, depends on the community health personnel. Taking note of these practices, majority have no 
Table 4. Table of correlations.

\begin{tabular}{lcc}
\hline Parameter & Correlation coefficient & Table value at $\mathbf{0 . 0 5}$ \\
\hline Health beliefs and compliance with medication & 2.24 & 2.05 \\
Health beliefs and compliance with out-patient referral & $3.31^{*}$ & 2.05 \\
Health practices and compliance with medication & 1.6 & 2.05 \\
Health practices and compliance with out-patient referral & $3.78^{\star}$ & 2.05 \\
\hline
\end{tabular}

Significant if computed coefficient is higher than the table value. * Significant at $p=0.05$.

have no significant risk posed to the community, however the need to review and revisit these practices is imminent in order to provide the best practices for them.

\section{Correlations}

Table 4 also revealed that there is no significant relationship between health beliefs and practices with compliance in medication which implies that whatever the type of respondents' beliefs and practices, compliance of medication is not affected. This is supported by Hunt (1997) that an individual whose concern is for his or her recovery and safety, regardless of his or her beliefs, appropriate measures will be provided or practiced to attain recovery, implying that as the severity of the illness increases the more, the person becomes more scientific of his or her beliefs and practices.

On the other hand, health beliefs and practices revealed a significant relationship with compliance of outpatient referral. According to Kuan (1993), compliance of the patient in clinic appointment is high if they are explained and taught on the significance of such intervention to the patient's recovery. In this study, it is found out that most respondents poorly complied with the follow up checkups because most of them believed that they are already recovered as they felt better and has not experienced any symptoms at all, which is as a result of lack of information of a person to the necessity of a particular intervention decreases compliance behavior. Poor compliance with discharge instructions were further investigated specially on out-patient referral. Respondents did not comply with the follow-up consultation since they do not feel any symptoms anymore or they went to a faith healer instead for further management.

\section{CONCLUSION AND RECOMMENDATIONS}

Based on the findings, health beliefs and practices do not influence compliance of medication since it is basically a consideration of individual's budgetary capacity. It is further concluded that health beliefs and practices significantly influence out-patient referral compliance since it has basically affected more on behavioral attributes rather than economic implications.

It is recommended that hospital administrators should formulate a standard patient-teaching guideline for patients to be discharged to provide guidance to patients' compliance of discharge instruction. Health personnel should also develop a working knowledge and nonjudgmental attitude towards' patients' health beliefs and practices to enable them to provide guidance and proper knowledge, especially those which are harmful to them.

\section{REFERENCES}

Berger K, Marilyn BW (1992). Fundamentals in nursing-collaborating for optimal health. Appleton \& Lange, Connecticut.

Hunt C, Agaton P, Richard C, Socorro E, Severino C (1997). Sociology in the Philippine setting. Phoenix Publishing House, Quezon City.

Kemp C (2005) Mexican \& Mexican-Americans: Health Beliefs \& Practices culture. Retrieved from https://bearspace.baylor.edu/Charles_Kemp/www/hispanic_health.ht m

Kaplan R (1993). Health and human behavior. McGraw Hill, Inc., New York.

Kuan L (1993). Effect of nursing strategies in compliance behavior of hypertensive individuals at UP-PGH. Vol. 2, No. 2, University of the Philippines Research Digest

Morisky D (2002). Traditional health beliefs, practices. Encyclopedia of public health.

Panopio I, Felicidad C (1995). Sociology (3rd ed.). Data on Morbidity. Sogod Rural Health Unit. Ken, Inc., Quezon City.

Mosot L (1985). Sogod Rural Health Unit. March-August 1985. Data on Morbidity. Unpublished report.

Neff $N$ (1998). Folk medicine in Hispanics in the Southwestern United States [Online], Retrieved: http://192.147.157.49/galaxy/Community/Health/FamilyHealth/Hispanic-Health.html

Vaughn L, Jacquez F. Baker R (2009). Cultural health attributions, beliefs, and practices: effects on healthcare. The Open Med. Educ. J. Retrieved:

http://www.benthamscience.com/open/tomededuj/articles/V002/SI001 6TOMEDEDUJ/64TOMEDEDUJ.pdf 